\title{
THE SUMMER MEETING IN BOULDER
}

The sixty-eighth Summer Meeting and the forty-first Colloquium of the American Mathematical Society was held at the University of Colorado, Boulder, Colorado, on Tuesday through Friday, August 27-30, 1963, in conjunction with meetings of the Mathematical Association of America, and the Society for Industrial and Applied Mathematics. The registration of the meetings was 1,305 , including 969 members of the Society.

Professor Saunders MacLane of the University of Chicago presented the Colloquium Lectures. The title of these lectures was Categorical algebra. Professor MacLane's first lecture was given at the opening session of the meeting, with the Society's president J. L. Doob, presiding. This session was held at 2:15 P.M. on Tuesday, August 27. The remaining three Colloquium Lectures were given at 9:00 A.M. on Wednesday, Thursday, and Friday, August 28-30. At these respective sessions, Professor MacLane was introduced by Professors Arne Magnus, Maurice Auslander, and Sardvadaman Chowla.

By invitation of the Committee to Select Hour Speakers for Annual and Summer Meetings, hour addresses were presented to the Society by Professor Sigurdur Helgason of the Massachusetts Institute of Technology, and by Professor Shlomo Sternberg of Harvard University. Professor Sternberg's address, entitled An algebraic model for transitive differential geometry, was given at 10:30 A.M. on Thursday, August 29. He was introduced by Professor A. M. Gleason. The title of Professor Helgason's talk was The Radon transform on symmetric spaces. Professor Helgason spoke at a session at 10:30 A.M. on Friday, August 30. He was introduced by Professor P. R. Halmos.

There were twenty-four sessions for contributed papers. The presiding officers at these sessions were Professor H. L. Alder, Professor R. V. Andree, Professor C. W. Austin, Professor J. R. Britton, Professor F. A. Ficken, Professor W. B. Fulks, Professor Frank Hahn, Professor G. C. Hewitt, Professor R. W. McKelvey, Professor J. E. Maxfield, Professor E. A. Michael, Professor L. J. Mordell, Professor H. T. Muhley, Dr. Dona Papert, Professor Everett Pitcher, Dr. H. O. Pollak, Professor G. B. Price, Dr. J. R. Rice, Professor R. W. Ritchie, Professor R. A. Rosenbaum, Professor E. C. Schlesinger, Professor Karl Stromberg, Professor R. R. Struik, and Professor J. E. Whitesitt.

Abstracts of the papers presented in person appear in the Notices of the American Mathematical Society for August, 1962.

A tea for participants and their families was presented on Tuesday, August 27, at 3:30 P.M. There was a barbecue on Wednesday, August 28 , at 5:30 P.M. 
The Committee on Arrangements for this meeting consisted of J. R. Britton, Chairman, H. L. Alder, W. E. Briggs, R. W. Ellingwood, T. C. Fry, C. A. Hutchinson, R. W. McKelvey, R. S. Pierce, W. E. Smith, L. C. Snively, and G. L. Walker.

The Council met on Tuesday, August 27, 1963, at 5:00 P.M.

The Secretary announced the election of the following one hundred forty-five persons to ordinary membership in the Society:

Abramson, Morton, McGill University;

Anderson, Ronald C., A. O. Smith Corporation, Milwaukee, Wisconsin;

Anton, Paul N., Lockheed Missile and Space Company, Sunnyvale, California;

Arquette, Lester K., Jr., Philco Computer Division, Willow Grove, Pennsylvania;

Bazar, David M., University of North Carolina;

Beekman, John A., University of Minnesota;

Behan, Donald F., University of Illinois;

Bennett, Carter L., New Mexico Highlands University;

Benzinger, Harold E., Syracuse University;

Bertuccelli, Harry C., Beckman Instruments/Systems Division, Fullerton, California;

Bierman, Gerald J., New York University;

Bleistein, Norman, New York University;

Borg-Costanzi, Edwin J., Royal University of Malta, Valletta, Malta;

Bossert, John M., University of Washington;

Brickman, Marjorie J., College of Arts and Sciences, Kumba, West Cameroon, Africa;

Brooks, John B., Nothing Music Company, New York, New York;

Buchman, Edwin O., California Institute of Technology;

Callahan, James J., New York University;

Cargo, David P., Wichita Falls, Texas;

Cassidy, Walter F., St. John's University;

Cavey, Edward F., Fordham University;

Chodrow, Mark, Harvard University;

Chuckrow, Vicki L., New York University;

Cooke, Robert W., Alameda High School, Alameda, California;

Cronin, Daniel D., Carleton College;

Daniel, Cuthrbert, New York, New York;

Diffie, Bailey Whitfield, Massachusetts Institute of Technology;

Distler, Raymond J., University of Kentucky;

Downs, Floyd L., Jr., Newton High School, Newtonville, Massachusetts;

Ferguson, Le Baron O., University of Washington;

Fischer, Gerd Otto-Walter, Mathematisches Institut der Universitaet, West Germany;

Fitzpatrick, Marjorie H., Auburn University;

Fortin, Jacques G., Laval University;

Fox, John H., Jr., Minneapolis-Honeywell Regulator Company, Hopkins, Minnesota;

Fung, Philip H. C., Case Institute of Technology;

Gilbert, Norma H., Fairleigh Dickinson University;

Gilford, Dorothy M., Office of Naval Research, Washington, D. C.;

Goeddel, Raymond J., United Air Lines, Inc., O'Hare International Airport, Chicago, Illinois;

Greenfield, Stephen J., Columbia University;

Greenwood, Joseph A., International Encyclopedia of the Social Sciences, New York, New York;

Greibach, Sheila A., Harvard Computation Laboratory, Cambridge, Massachusetts; 
Hailpern, Raoul, Park School of Buffalo, Buffalo, New York;

Hall, Mary C., Lowell Technological Institute, Lowell, Massachusetts;

Hall, Michael H., University of Arizona;

Holland, Paul W., Stanford University;

Howard, Charles M., University of California, Berkeley;

Howson, Joseph T., Jr., Rensselaer Polytechnic Institute;

Itzkowitz, Gerald L., University of Rochester;

Jeske, James A., Ames Research Center, National Aeronautics and Space Administration, Moffett Field, California;

Johnson, Ben, Oregon State University;

Kalin, Theodore A., Air Force Cambridge Research Laboratories, Cambridge, Massachusetts;

Kenney, Margaret J., Boston College;

Kim, Jehpill, Chung-chong University, Taejon, Korea;

Kleiman, Steven L., Harvard University;

Knight, Ronald A., Brigham Young University;

Kolker, Robert J., Minneapolis-Honeywell Regulator Company, Boston, Massachusetts;

Krajkiewicz, Paul M., Illinois Institute of Technology;

Kretschmer, Kenneth S., General Electric Company, New York, New York;

Kymala, Earl E., University of Southern California;

Leary, James M., New York University;

Livingston, William R., University of Illinois;

Luh, Johnson Y. S., International Business Machines Corporation, Rochester, Minnesota;

McCarthy, Donald J., New York University;

McGehee, Oscar C., 2774 Yale Station, New Haven, Connecticut;

McGrath, John B., Bell Telephone Laboratories, Whippany, New Jersey;

McKibben, William P., Georgia Institute of Technology;

McLemore, Ethel W., Dallas, Texas;

MacKichan, Alan K., K. B. MacKichan and Associates, Grand Forks, North Dakota;

Maloney, John P., U. S. Government, Warrenton Training Center, Warrenton, Virginia;

Maranto, Frank A., Science Information Exchange, Washington 9, D. C.;

Marcus, Curtis F., System Development Corporation, Santa Monica, California;

Maxwell, J. Glenn, Ohio State University;

Mayoh, Brian H., University of Illinois;

Miller, Penelope A., Aerosystems Company, Buffalo, New York;

Morgado, Jose C., Jr., Inst. of Physics and Math., Recife, Pe., Brazil;

Morrison, Laura M., Esso Research and Eng., Florham Park, New Jersey;

Morrow, James A., California Institute of Technology;

Musielak, Julian, University of Poznan;

Nath, Radha G., Utah State University;

Normand, Gerard L., College Militaire Royal;

Olano, Calvin A., Jr., Gretna, Louisiana;

Olson, Vivienne H., Portland State College;

Orr, Richard J., Case Institute of Technology;

Paige, Barbara J., Ampex Corporation, Redwood City, California;

Pareigis, Bodo, 3414 Hardegsen, Vor dem Tore 5, Germany;

Patterson, James W., Central Methodist College;

Pfeffer, Allen M., California Institute of Technology;

Pflugfelder, Hala O., Temple University; 
Prendergast, Hugh Brent, University of Manitoba;

Pyles, Warren S., U. S. Patent Office, Washington, D. C.;

Quinn, John Francis, Pratt and Whitney Aircraft Company, West Palm Beach, Florida;

Randall, Charles H., Knolls Atomic Power Laboratory, General Electric Company, Schenectady, New York;

Raspa, Peter D., Montreal, Quebec, Canada;

Ratib, Ismail, sh. Ein Shames, Ezbet El Nakhl, Cairo, Egypt, UAR;

Rhoads, Donald H., Andrews University;

Richmond, Allan E., Tektronix, Incorporated, Beaverton, Oregon;

Riney, John S., Research Analysis Corporation, Bethesda, Maryland;

Roberson, Clifford J., Jr., New Mexico State University;

Robertson, Harold G., University of Kentucky;

Rod, David L., University of Wisconsin;

Rooff, Karen L., Control Data Corporation, Newark, New Jersey;

Rosencrans, Steven I., Massachusetts Institute of Technology;

Rosenthal, Peter M., University of Michigan;

Ross, Bertram, Wilkes-Barre, Pennsylvania;

Rouse, Herbert R., Tufts University;

Ruggiero, Rocco R., Republic Aviation Corporation, Farmingdale, New York;

Saccomanno, Mary E., Wright Jr. College;

Saenger, Rudi F., Navy Oceanographic Office, Washington 25, D. C.;

Said, Turki M., Ramtha-Turra, Jordan;

Schultz, Martin H., Harvard University;

Saksena, Gajraj B., North American Aviation Incorporated, Downey, California;

Sarapuka, Peter K., Illinois State Geological Survey, Urbana, Illinois;

Scribner, Terry L., United Aircraft Corporation, East Hartford, Connecticut;

Seaman, Donna J., Sylvania Electronic Defense Laboratory, Mountain View, California;

Seifer, Arnold D., Rensselaer Polytechnic Institute;

Sidney, Stuart J., Harvard University;

Simon, Dr. Hermann, McGill University;

Sinha, Chandreshwar P., Bihar Institute of Technology, Bihar State, India;

Sister Marie Genevieve, Mount Saint Mary College;

Sister Mary Stephenie, Georgian Court College;

Skinner, Charles E., Jr., International Business Machines Corporation, Kingston, New York;

Smith, Don R., U.S. Naval Ordnance Laboratory, White Oak, Silver Spring, Maryland;

Sobel, Milton, University of Minnesota;

Sprecher, David A., University of Maryland;

Stark, Thomas I., Chicago City Jr. College;

Stone, Alexander P., University of Illinois;

Stump, Robert K., Muhlenberg College;

Suchar, Joseph T., Villanova University;

Tellefsen, Carl R., Purdue University;

Tooth, Clive J., City of London School, London, England;

Tyree, Alan L., University of Washington;

Uno, Michio, General Precision Incorporated, Glendale, California;

Valdivia, Oscar, Centro de Investigacion del I P N, Mexico, D. F., Mexico;

Van Akin, Everett F., New York University College;

Wallace, Henry G. S., Jr., General Electric Company, Bethesda, Maryland; 
Wallace, William, Bronx Community College;

Weinbaum, Carl M., New York University;

Wheaton, Burdette C., State University of Iowa;

White, Harry J., Portland State College;

Willig, Paul M., New York University;

Wilson, Raymond B., Brown University;

Winslow, Richard E., Columbia University;

Wood, James T., University of Pennsylvania;

Woods, William A., Jr., Ohio Wesleyan University;

It was announced that the following had been admitted to the Society in accordance with reciprocity agreements with various mathematical organizations:

Sociêté Mathématique de Belgique: Richard W. Feldman.

Berliner Mathematische Gesellschaft: Klaus Habetha.

Calcutta Mathematical Society: A. Satyanarayana Rao.

Dansk Matematisk Forening: Christian M. Gram.

Deutsche Mathematiker Vereinigung: Hermann Heineken.

Sociêté Mathématique de France: Marie-Paule Brameret, Rene Emard, Philippe Noverraz, Andre L. Peltier, Louis G. Robin, Ngo Van Que.

Gesellschaft fiur Angewandte Mathematik und Mechanik: Siegfried H. Lehnigk.

Indian Mathematical Society: Dhupa R. Bajracharya, Shankar H. Dwivedi, Syed M. Mazhar, J. Sudarsana Rao, Vemuri L. N. Sarma, S. S. Subramanyam.

Mathematical Society of Japan: Koji Honda, Tsuneo Kanno, Shoji Koizumi, Noboru Suzuki.

London Mathematical Society: Barry W. Bugden, Lionel W. Longdon, Alun O. Morris, Ohn E. Peters.

Malayan Mathematical Society: Kua Ee Kia, Peter Lancaster, Kok L. Ng.

Svenska Matematikersamfunder: Karl Greger.

The following thirty-four persons have been elected to membership in the Society on nomination of institutional members as indicated:

Antioch College: John T. Cannon, Julian Gevirtz, Jean E. Ross.

Emory University: Dorothy S. Rutledge, John M. Saade, John St. Clair Werth, Jr.

Georgia Institute of Technology: Roy Bushnell Bogue, George L. Cain, Jr., Alan G. Law.

Johns Hopkins University: Courtney W. Doyle, Marvin H. J. Gruber, Arnold S. Kas,

Janet E. Lacetera, Bernard B. Morrel, Sanford M. Orlow, Bruce D. Schaeffer,

Robert J. Yankevitch, Elaine E. Yarow.

Oregon State University: Charles B. Balogh, Mason E. Henderson.

San Fernando Valley State College: Irving S. Florman, Marvin Schaefer.

Swarthmore College: Dorothy W. Wolfe.

Syracuse University: Paul Abramson, David T. Brown, Margaret M. Humm, Thomas V. O'Brien.

William Marsh Rice University: Karl F. Barth, Jack D. Bryant, Robert L. Hall, John E. McMillan.

Xavier University: Benito J. Cerimele, Robert F. Cissell, David C. Flaspohler.

The following Presidential appointments were reported:

As a Joint Committee on Graduate Program in Mathematics: C. E. Rickart, Chairman pro tem, David Blackwell, Eldon Dyer, S. Eilen- 
berg, M. K. Fort, Jr., P. D. Lax, Ivan Niven, A. D. Wallace, G. T. Whyburn.

To the Committee on Summer Institutes: P. T. Bateman, Elias Stein, Leonard Gillman, Chairman.

As a Committee to Study the Financial Problems of Mathematical Reviews: E. F. Beckenbach, Chairman, Andrew Gleason, N. Kazarinoff, Marston Morse, D. C. Spencer, John Wehausen.

As a Committee to advise on the National Register: Robert Rinehart, S. S. Wilks, H. M. Gehman, M. M. Flood, Barkley Rosser.

As a Committee on the Chinese Dictionary: S. H. Gould, Chairman, S. T. Hu, H. C. Wang.

The Secretary reported that the following persons had accepted invitations to deliver hour addresses at meetings of the Society: Sigurdur Helgason and Shlomo Sternberg at Boulder, Colorado, August, 1963. Louis de Branges at the University of Wisconsin, November, 1963.

The Secretary reported that the following members represented the Society: T. Freeman Cope at the inauguration of Dr. Dumont F. Kenny as President of Queensborough Community College of the City University of New York; Robert Steinberg at the 1963 meeting of the Italian Mathematical Union; Elliott L. Buell at the inaguration of Clifton Woodford Emery, Jr. as President of Worcester Junior College.

The following meetings of the American Mathematical Society were set: New York University, February 29, 1964; Reno, Nevada, April 28, 1964.

The Council elected to Institutional membership the University of Montreal and elected the McGraw-Hill Book Co. as an Institutional Associate.

There was a business meeting of the Society at 10:30 A.M. Wednesday, August 28 in Macky Auditorium. The Secretary read a report on recent activities and present problems of the Society; his report is to be found in the October issue of the Notices. Professor Herman Rubin brought up the point that at the present meeting, some papers had been scheduled to conflict with a major social event, and suggested that consideration be given to the possibility of scheduling evening sessions. There followed a considerable discussion of the desirability of this, and of the general problem of scheduling a large number of activities without conflict.

JOHN W. GREEN

Secretary

R. S. PierCe

Associate Secretary 